\title{
Zum Jubiläum des Kongresses für innere Medizin.
}

Von Prof. H. Strauss in Berlin.

25 Jahre sind am 20. April d. J. vergangen, seitdem v. Frerichs den ersten Kongreß für innere Medizin in Wiesbaden eröffnet hat. Fast 200 Aerzte kamen damals aus dem Süden und Norden Deutschlands zusammen, um „sich zu verständigen über Fragen, welche die deutsche Heilkunde bewegen, um Erfahrungen. auszutauschen, Ideen anzuregen und auch auszuführen und die gemeinsamen Interessen zu vertreten". Seit diesem ersten Kongrel3 hat sich gar Manches nicht $b l o ß$ in der äußeren Erscheinung des Kongresses, sondern auch in den Fragen geändert, die den Kongreß und die innere Medizin beschäftigen. Ein alter - glücklicherweise recht beträchtlicher - Stamm ist zwar geblieben, der die Traditionen jener Zeit der jüngeren Generation überliefert, es fehlen aber doch - um nur von den Rednern des I. Kongresses zu sprechen - Männer wie v. Frerichs, Gerhardt, Nothnagel, Liebermeister, Riegel, Rosenstein, Rühle, Immermann, Seitz u. a., Männer, die nicht bloß durch ihr Wissen und Können, sondern auch durch ihre Persönlichkeit dazu beigetragen haben, dem ersten Kongreß für innere Medizin eine Gestalt zu geben, die für die Zukunft grundlegend war.

Wer hätte vor 25 Jahren geahnt, welchen Umfang und welche Entwicklung die innere Medizin in den folgenden 21/2 Dezennien nehmen würde und welche Aufgaben damit dem Kongreß erstehen würden? Wer hätte speziell damals den Aufschwung prophezeien können, den seither die Lehre von den Infektionskrankheiten genommen hat, und gar an die Entstehung ganz neuer Disziplinen auf diesem Gebiet, wie der Serotherapie und Serodiagnostik gedacht? Wer hätte sich weiter die Fortschritte träumen lassen, welche seither die physikalische Diagnostik durch die Röntgenstrahlen und die chemische Diagnostik durch die Entwicklung der medizinischen Chemie erfahren hat, und wer hätte sich ein Bild von dem Einfluß machen können, welchen die Fortschritte der Technik auf die Therapie, speziell auf die physikalische Therapie - man denke nur an die Lichttherapie -, inzwischen gewonnen hat? Wer hätte schließlich auf dem ersten Kongreß, auf welchem "ein Fall von gelungener 
Resektion eines carcinomatösen Pylorus" als Seltenheit demonstriert wurde, daran gedacht, da $\beta^{\circ}$ es schon wenige Jahre später "Grenzgebiete $^{\star}$ geben würde, die zu eifrigen Diskussionen und zu heißen Kämpfen führen würden. Nach gar vielen Richtungen hin haben sich also Interessenkreis und Aufgaben des Kongresses für innere Medizin in den verflossenen $21 / 2$ Jahrzehnten erweitert. Schon vor 25 Jahren hat v. Frerichs geklagt, wie sehr man sich von der durch die innere Medizin vertretenen Einheitsidee des menschlichen Organismus entferne, und hervorgehoben, wie sehr die innere Medizin berufen sei, diese Einheitsidee festzuhalten und auszubauen. Heute muß diese Mission der inneren Medizin noch schärfer betont und eindringlicher ausgesprochen werden, wo die Neigung zur Zersplitterung und zur Abzweigung von Spezialitäten noch größer geworden ist, als damals. Dazu hat noch die rapide Entwicklung der verschiedenen z. Zt. noch im engsten Verhältnis zur Mutterdisziplin stehenden Tochterdisziplinen im Laufe der Zeit eine solche Fülle wissenschaftlicher Arbeiten gezeitigt, daß diesen Arbeiten gegenüber die strengste Kritik und Auslese angebracht erschien, was an sich schon eine Zentralstelle für eine gemeinsame Aussprache notwendig macht. Auch die sogenannten Hilfswissenschaften haben in den letzten Jahrzehnten einen so gewaltigen und für die innere Medizin so ergebnisreichen Aufschwung genommen, daß auch hier ebenso sehr, als es schon v. Frerichs betont hat, auch heute noch für die Klinik Veranlassung vorliegt, das Material kritisch zu sichten und es auf seine Uebertragbarkeit auf die praktische Medizin sorgfältig zu prüfen. Neue Aufgaben sind -der inneren Medizin auch durch die moderne soziale Gesetzgebung erwachsen, die eine Menge neuer Fragen für Diagnostik und Therapie angeregt hat.

Bis zu welchem Grade der Kongreß für innere Medizin diesen Aufgaben gerecht geworden ist, lehrt ein Blick auf die bisher erschienenen 23 Bände der Verhandlungen. Der Kongreß hat nicht bloß zu zahlreichen, die medizinische Welt bewegenden Zeit- und Streitfragen durch mustergültige Referate und eingehende Diskussionen berufenster Beurteiler klärende Stellung genommen, sondern auch dadurch, da $\beta$ er zur Mitteilung einer Reihe wichtiger neuer Entdeckungen und Beobachtungen Gelegenheit gab, zu der großen Entwicklung beigetragen, welche die innere Medizin in den letzten Jahrzehnten genommen hat. Wie vielseitig der Kongreß für innere Medizin seine Aufgaben betrachtet hat, ergibt sich am besten durch einen Blick auf die offiziellen Referate der 23 Zusammenkünfte des Kongresses. ${ }^{1}$ )

Am instruktivsten ist vielleicht nach dieser Richtung hin cine Betrachtung der sich auf die Infektionskrankheiten erstreckenden Themata und ganz besonders ein Vergleich der dem Kapitel "Tuberculose“ gewidmeten Referate. Als auf dem 1. Kongreß für innere Medizin der Regierungsrat Dr. Robert Koch vier Wochen nach seiner ersten Mitteilung über diesen Gegenstand einen Vortrag über die "Aetiologie der 'Tuberculose" hielt, konnte sich noch niemand vorstellen, daß zehn Jahre später die von Curschmann, v. Jaksch, Ziegler, Heubner, Moritz Schmidt, Dettweiler, Sonnenburg, Baccelli, Klebs über "das Kochsche Heilverfahren bei Lungentuberculose" eingeleitete Debatte die Blicke der ganzen gebildeten Welt auf sich lenken würde; eine Debatté, deren reservierte Haltung gegenüber dem Verfahren leider auch heute kaum viel anders ausfallen würde, so gerne auch jedermann zugeben wird, daß die Entdeckung des großen Meisters seither der Prophylaxe sichere Wege gewiesen hat. Kaum einer Einschränkung bedürfen auclı heute noch die Schlüsse einer auf dem VI. Kongreß von Dettweiler und Penzoldt eingeleiteten Debatte über "die Therapie der Phthise", in welcher sich die verschiedenen Redner (auch Brehmer war noch unter ihnen) für die hohe Bedeutung des physikalisch-diătetischen Heilverfahrens aussprachen.

Nicht minder interessant als die Tuberculose-Debatten waren auch die Diskussionen über die Diphtherie, die auf dem II. Kongreß von Gerhardt und Klebs vorwiegend nach der anatomischklinischen Seite geführt wurden, und bei welcher Gelegenheit Heubner, der auf einem späteren (XIII.) Kongreß über dasselbe Gebiet als Referent fungierte, in der Diskussion noch sagen konnte: ,das, wie auch ich glaube, aus organisiertem Material bestehende Diphtheriegift ist zurzeit noch nicht gefunden". Als $\mathrm{He} \mathrm{ubner}$ ein Jahrzehnt später über die Heil- und Schutzwirkung des Behringschen Heilserums referierte, war die Erörterung allerseits von einer hoffnungsvollen Stimmung getragen, und mit Freuden können wir heute feststellen, daß das Vertrauen, welches die weitaus überwiegende Mehrzahl der Diskussionsredner diesem Mittel entgegenbrachte, in der Folgezeit nicht getäuscht wurde. Auch die Cholera war zweimal Gegenstand der Diskussion, und zwar auf dem

1) Im Jahre 1903 fiel der Kongreß wegen des Internationalen medizinischen Kongresses in Madrid aus.
VII. KongreB im Anschlu $B$ an ein Referat von $P$ feiffer und Cantani und auf dem XII. Kongreß im Anschluß an ein durch die damalige Hamburger Epidemie veranlaßtes Referat der derzeit berufensten Beurteiler der Krankheit, $R$ u $m$ pf und $G$ a f $\mathrm{k} \mathrm{y}$. Von sonstigen Infektionskrankheiten bildeten noch auf dem VI. Kongreß eine durch Vogel und Hagenbach eingeleitete Erörterung über die. Pathologie und Therapie des Keuchhustens, sowie auf dem V. Kongreß ein Referat von Kaposi und Neisser über die Therapie der Lues den Gegenstand eines Referats. Wie würde sich wohl der letztgenannte Referent heute, wo wir die Spirochäten kennen und den Affen als Objekt für experimentell-therapeutische Versuche besitzen, äber dasselbe Thema äußern? Auch das Fieber und die Antipyrese bildeten zweimal - und zwar auf dem IV. Kongreß durch Filehne und Liebermeister und auf dem XV. Kongreß durch Kast und Binz den Gegenstand eines Referats, und es hat Binz außerdem noch auf dem VII. Kongreß im Verein mit v. Jaksch über die auch in neuerer Zeit wieder mehrfach erörterte Frage, "Weingeist als Heilmittel“, referiert.

Dem Kapitel der Infektionskrankheiten steht auch das auf dem III. Kongreß von $\mathrm{Jürgensen}$ und A. Fränkel erstattete Referat über die genuine $\mathrm{P}$ neumonie nahe, das einen besonderen Reiz durch die Ausfïhrungen A. Fränkels über den damals noch strittigen Infektionserreger gewann. Auch nach der therapeutischen Seite stand die Pneumonie - auf dem XVIII. KongreB - im AnschluB an ein Referat von F. v. Korányi und Pel zur Diskussion. Ueber die operative Behandlung der Pleuraexsudate wurde auf dem V. Kongreß ron Fraentzel und Weber und über die Behandlung der Empyeme auf dem IX. Kongreß von Immermann und Schede referiert. Mustergültig und anregend war auch das von Curschmann und Riegel auf dem IV. Kongreß erstattete Referat über das Bronchialasthma.

Besonders ausgiebig beschäftigte sich der Kongreß in seinen offiziellen Referaten mit den Erkrankungen des Herzens und der Nieren. Von den ersteren war besonders interessant das auf dem VII. Kongreß erstattete Referat von Oertel und Lichtheim über die Behandlung chronischer Herzmuskelkranken, und zwar nicht bloß wegen der damaligen und noch lange Zeit später vorhandenen Aktualität des Gegenstandes, sondern auch aus dem Grunde, weil Li ch theim in seinem Referat gegenüber den Oertelschen Theorien in so klarer Weise seine reservierte Haltung begründete, daß diese auch bei weiterer Prüfung der Frage die herrschende Auffassung blieb. Eines der wichtigsten Referate auf dem Gebiete der Herzkrankheiten war auch das auf dem X. Kongreß gehaltene, von A. Fränkel und O. Vierordt über die Angina pectoris. Ein tragisches Feschick brachte es mit sich, daß der zuletzt genannte Autor gerade dieser Krankheit zum Opfer fiel Der Endocarditis und ihren Beziehungen zu anderen Krankheiten widmete Litten auf dem XVIII. Kongreß ein ebenso gründliches als klares Referat. Hervorragend instruktiv und praktiscl wichtig waren auch drei in gewisser Beziehung zusammengehörige Referate, nämlich das auf dem XVII. Kongreß von Martius und von v. Schrötter entwickelte Referat über die Insuffizienz des Herzmuskels und das auf dem XIX. Kongreß von Gottlieb und Sahli erstattete Referat über Herz- und Vasomotorenmittel, sowie das auf dem XXI. Kongreß gehaltene Referat von Marchand und Romberg über Arteriosklerose. Konnte man aus den genannten Referaten ersehen, welche Fortschritte die moderne, mehr funktionelle Betrachtung im Verein mit den Ergeb nissen der experimentellen Pathologie für die Therapie der Herz krankheiten gebracht hat, so unterrichtete uns ein anderes Referat, nämlich das auf dem XXIII. Kongreß von Hering über die Un regelmäßigkeiten des Herzens erstattete, über die Vertiefung, welche unsere Kenntnis des Mechanismus der Herztätigkeit unter physiologischen und pathologischen Bedingungen in den letzten Jahrzehnten gewonnen hat. Die Pathologie und Therapie des Morbus Brightii war zweimal Gegenstand von ausgezeichneten Referaten, und zwar auf dem I. Kongreß durch v. Leyden und Rosenstein und auf dem IX. KongreB durch Senator und v. Ziemssen. Das Senatorsche Referat könnte heute nach $11 / 2$ Jahrzehnten mit nur selır geringfügigen Aenderungen noch in derselben Form gehalten werden.

Etwas spärlicher als die oben genannten Krankheitsgruppen waren die Erkrankungen des Verdauungskanals unter den Referaten vertreten. Unter diesen erregte seinerzeit ein besonderes Interesse das auf dem III. Kongreß gehaltene Referat von Le ube und Ewald über die nervöse Dyspepsie. Ein zweites Mal fun gierte Ewald zusammen mit Fleiner auf dem $\mathrm{XX}$. Kongreß für innere Medizin als Referent über die Diagnose und Therapie des Magengeschw ürs. Von besonderer Bedeutung war auf dem vorliegenden Gebiet das den "Grenzgebieten" angehörende, auf dem VIII. Kongreß von Leichtenstern und Curschmann gehaltene Referat über den Ileus und seine Behandlung, sowie das aul 
dem XIII. Kongreß von Sahli und Helferich erstattete Referat über die Pathologie und Therapie der Typhlitiden. Man kann beide Referate auch heute noch, wo sich die Anschauungen nach verschiedenen Richtungen hin geändert haben, mit großem Genuß und mit reichem Gewi in an Kenntnissen lesen. Zeichnete sich doch das erstere trotz seiner für heutige Begriffe etwas zu weit gehenden Reserve gegenüber der Chirurgie durch eine bewundernswerte Klarheit der Darstellung des überaus komplizierten Gegenstandes aus, und zeigte doch das zweite - in unserer heutigen Zeit, in der an so vielen Stellen ein das Denken überflüssig machender Schematismus auf dem vorliegenden Gebiete zu herrschen beginnt $\mathrm{da} \beta$ eine kritisch-individualisierende Indikationsstellung auch be der Behandlung der Blinddarmentzündungen Erfolge zu zeitigen vermag. Von den sonstigen Referaten aus dem Gebiete der Verdauungspathologie verdiente und fand auch besondere Beachtung dasjenige von Friedrich Müller und Brieger (XVI. KongreB) über die Autointoxikationen intestinalen Ursprungs, weil beide Referenten an gar manchen Stellen nüchterne Kritik an die Stelle phantasiereicher Deduktionen gesetzt haben. In hervorragendem Maße klärend hat auch das lichtvolle, auf dem X. Kongreß gehaltene Referat über Gallensteinkrankheiten von Naunyn und $\mathrm{F}$ ürbringer gewirkt. Ebenso hat auch das auf dem XI. Kongreß gehaltene Referat von Rosenstein und Stadelmann über chronische Leberentzündung die besprochene Frage außerordentlich gefördert.

Große Anregung und großen Gewinn hat auch das Gebiet der Blut- und Stoffwechselkrankheiten von den Debatten des Kongresses gehabt. Ehrlich selbst hat im Vcrein mit BirchHirschfeld auf dem XI. Kongreß in einem Referat über schwere an ämische Zustände das Wort ergriffen und dort seine heute noch entscheidenden Auffassungen über die Bedeutung der mor photischen Blutveränderungen bei schweren anämischen Zuständen entwickelt. Auf dem XVII. KongreB kam es im AnschluB an ein Referat von Loewit und Minkowski über die Leukämie und Leukocytose zu einer heftigen Debatte, die zu einer Ablehnung der Loewitschen Parasiten geführt hat. Von praktisch therapeutischer Wichtigkeit war auch das auf dem XIII. Kongreß von Bunge und $Q u i n c k e$ erstattete Referat über Eisentherapie. Von den Erkrankungen des Stoffwechsels stand auf dem IV. Kongreß ein Re ferat von Ebstein und Henneberg über Fettleibigkeit und auf dem V. ein solches über den Diabetes von Stockvis und F. A. Hoffmann zur Diskussion. Außerdem erstattete Leo auf dem XVI. KongreB ein umfassendes Referat über die Behandlung des Diabetes. Die zunehmende Erforschung des Gasstoffwechsels und die organotherapeutischen Bestrebungen, welche vor nicht zu langer Zeit in der Medizin Platz griffen, zeitigten drei Referate, die sich auf die Pathologie der Schilddrüse bezogen, nämlich das auf dem XIV. Kongreß von Ewald und Bruns entwickelte Referat über die therapeutischeAnwendung derSchilddrüsenpräparate, das auf dem XV. Kongreß erstattete Referat von Eulenburg über Morbus Basedowii und das auf dem letzten (XXIII.) Kongreß gehaltene Referat von F. Kraus und Kocher über die Pathologie der Schilddrüse. Das ebenso geistvolle als inhaltsreiche und auf dem Boden zahlreicher eigener Untersuchungen kritisch gehaltene Referat von $\mathrm{Kraus}$ zeigte nicht nur, wie viel Neues auf dem Gebiete der Physiologie und Pathologie der Schilddrüse durch emsigen Forscherflei $\beta$ in einem Jahrzehnt produziert worden ist, sondern auch wieviel neue Fragen für die Klinik und für die experimentelle Pathologie gerade durch diese Arbeiten aufgeworfen worden sind. In der Anwendung der Thyroideapräparate hat sich, wenn wir den Blick rückwärts wenden, allerdings in den zehn Jahren, die seit dem Ew ald schen Referat verflossen sind, trotz unveränderter Anerkennung der Wirkung der Thyreoideasubstanz eine sehr berechtigte Zurückhaltung bemerkbar gemacht.

Von den Erkrankungen des Nervensystems standen die Lokalisation der Gehirnkrankheiten im Anschlub an ein Referat von Nothnagel und Naunyn auf dem VI. KongreB, die Frage der Epilepsie im AnschluB an ein Referat von Unverricht auf dem XV. Kongreß und die Frage der Myelitiden im Anschluß an ein Referat von v. Leyden, Redlich und v. Strümpell auf dem XIX. Kongreß zur Diskussion. Auf dem XII. Kongreß referierten v. Strümpell und Wernicke über das durch die moderne soziale Gesetzgebung so wichtig gewordene Kapitel der trau. matischen Neurosen. Aber auch Fragen medizinisch-sozialer Natur beschäftigten den Kongreß, wie sich dies bei der auf dem $X V I$. Kongre $\beta$ im Anschlu $\beta$ an das Referat von v. $\mathrm{Zi}$ emssen und v. Jaksch erfolgten eingehenden Diskussion des medizinischen Unterrichts zeigte.

Aus dem Gebiete der physikalischen Therapie wurde auf dem $X X$. Kongreß von $B i$ e ein Referat über die zu so großer Bedeutung gelangte Lichttherapie gehalten, und es wurde die physikalische Therapie auch bei Gelegenheit des auf dem XV. Kon- greß gehaltenen Referats von $\mathrm{Ba}$ eumler und $\mathrm{O}$ t $\mathrm{t}$ über den chronischen Gelenkrheumatismus gestreift, bei welchem Anlaß auch eine rege Diskussion über die Pathogenese des chronischen sowie des akuten Gelenkrheumatismus stattfand. Mit dem Dispositionsbegriff hatte sich der Kongreß schon während seiner sechsten Tagung bei Gelegenheit der Diskussion äber die Therapie der Phthisis beschäftigt, aber noch eingehender wurde dieser alte durch die neuesten Forschungen der Bakteriologie wieder zu Ehren gebrachte Begriff bei Gelegenheit eines auf dem XXII. Kongreß von Ziegler und Martius erstatteten Referats über die Vererbung erörtert.

Diese kurze Uebersicht zeigt, wie vielseitig die offiziellen Diskussionsthemata während des 25 jährigen Bestehens des Kongresses für innere Medizin waren, und wie viele Zweige der inneren $\mathrm{Me}$ dizin aus seinen Tagungen Nutzen gezogen haben. Leider ist es nicht möglich, in dem kurzen, hier gesteckten Rahmen auch nur anzudeuten, wie weit das letztere auch durch die auf den verschiedenen Kongressen gehaltenen Einzelvorträge und durch die von ihnen veranlaßten $D$ iskussionen der Fall war. Wie sehr die innere Medizin aber auch durch diese Vorträge gefördert wurde, ergibt sich aus einem kurzen Hinweis auf einige wenige Vorträge, so z. B. auf die Mitteilungen von v. Mering über den Phloridzin-Diabetes (V. Kongreß), von Minkowski über Diabetes nach Pancreasexstirpation (XI. Kongreß), von v. Noorden über die Behandlung harnsaurer Konkremente mit Kalk (XIV. Kongreß), von Quincke über die Lumbalpunktion (X. KongreB), von Wenkebach über die physiologische Erklärung der Arhythmie des Herzens (XVIII. Kongreß), von Moritz über die Ergebnisse der Orthodiagraphie für die Herz untersuchung (XIX. Kongreß), sowie auf die auf dem XV. Kongre $B$ gehaltenen Vorträge von v. Behring über experimentell begründete ätiologische Therapie und von Liebreich über die Ziele der modernen medikamentösen Therapie, und viele andere wichtige Vorträge, von welchen hier nur noch die auf dem XXII. KongreB gehaltenen Vorträge über die Röntgenbehandlung der Leukämie speziell genannt sein mögen.

Große Bedeutung haben manche Referate und Vorträge - das soll besonders betont werden - gerade durch die Anregung von Diskussionen erlangt, in denen oft die erfahrensten Kenner des betreffenden Gebiets den Niederschlag ihrer langjährigen Erfahrungen der Oeffentlichkeit übergaben. Ist doch gerade für die Beurteilung klinischer Fragen, bei deren Betrachtung das subjektive Moment eine so große Rolle spielt, die Meinung möglichst zahlreicher, mit recht verschiedenen Gesichtspunkten an die Frage herantretender Beurteiler erwünscht und zwar besonders, wenn die Beobachtungen unter recht verschiedenartigen Bedingungen gemacht sind.

So sehen wir denn, wenn wir rückwärts schauen, daß der Kongre $\beta$ die innere Medizin nach vielen Richtungen hin gefördert und bereichert hat und $\mathrm{da} ß$ wir an seinem Jubeltage berechtigten Grund haben, mit stolzer Genugtuung auf die vergangenen 25 Jahre zurückzublicken. Es hieße aber undankbar sein, wenn wir uns bei einer Betrachtung, welcher den wissenschaftlichen Ergebnissen des Kongresses gilt, nicht auch der schönen, das Gemüt erfreuenden, Stunden erinnerten, welche die einzelnen Kongreßorte den Teilnehmern bereitet haben. $14 \mathrm{mal}$ tagte der Kongreß an dem Ort seiner Gründung, der schönen, meist im üppigen Frühjahrsschmuck prangenden Taunusstadt, deren Reize bei vielen Mitgliedern desKongresses, namentlich den im Norden wohnenden, die Reiselust steigerten, wenn diese durch die vom Kongreß in Aussicht gestellten Darbietungen schon geweckt war. Trotzdem zog der Kongreß neunmal aus und zwar dreimal nach Berlin, je zweimal nach $M$ ü $\mathrm{nch}$ en und Leipzig und je einmal nach Wien und Karlsbad. Auch an diese Städte knüpfen sich für die Besucher angenehme Erinnerungen, aber es wird sich doch auf dem diesjährigen Kongreß zeigen, bis zu welchem Grade die Reize von Wiesbaden überwiegen.

Jedenfalls haben die "Reisen" des Kongresses eine gewisse Abwechslung nicht nur in das äußere Bild, sondern auch in die Dar bietungen der einzelnen Kongresse insofern gebracht, als sie in den betreffenden Städten die Gelegenheit zur Kenntnis besonderer medizinischer Einrichtungen und Anstalten gegeben hat. Noch mehr aber als durch diesen Faktor wurde die "Individualität" der einzelnen Kongresse durch die Persönlichkeit des jeweiligen Kongre $B$ leiters bestimmt. Bei den drei ersten Tagungen führte noch v. Frerichs den Vorsitz, dann lag die Leitung viermal in der Hand von v. Leyden und je einmal in der Hand von Gerhardt, v. Leube, Liebermeister, Nothnagel, Curschmann, Immermann, v. Ziemssen, Baeumler, Moritz Schmidt, Quincke, v. Jaksch, Senator, Naunyn, Merkel, Erb und v. Strümpell. Viele von diesen Vorsitzenden haben in ihren Eröffnungsreden Worte gesprochen, die eines dauernden Platzes in den Ueberlieferungen der Medizin wert sind. Von den einzelnen Kongreßleitern hat sich aber kaum einer so sehr um die Entwicklung und das Gedeihen des Kongresses verdient gemacht, wie v. Iueyden, 
der seit 11/2 Jahrzehnten konsultierendes Mitglied des Geschäftskomitees ist und von dem Naunyn gelegentlich der auf dem $X X$. Kongre $B$ begangenen Feier des 70 . Geburtstages v. Leydens sagte: „Ich übertreibe keineswegs, wenn ich unseren Kongreß als Leydensche Schöpfung bezeichne. Jeder, der unsere Entstehungsgeschichte kennt, weiß, daß v. Leyden ihn ins Leben gerufen hat, und jeder, der unserer Entwicklung gefolgt ist, weiß, daß v. Le yden die Seele dieses Kongresses gewesen ist, von Anfang bis heute." Gewiß verdankt der Kongreß seine Stellung den Leistungen aller seiner Mitarbeiter, es markieren sich aber doch diejenigen seiner Eührer besonders, und es darf deshalb mit besonderer Freude begrüßt werden, daB von seinen Gründern und Führern noch so viele an seinem Jubiläum teilnehmen. Möge es diesen vergönnt sein, den Kongreß noch recht häufig zu besuchen und damit der jüngeren Generation einen Ansporn zu geben, den Kongreß in den alten Bahnen weiterzuführen. Mögen aber auch die kommenden Zeiten so viele Entdeckungen bringen, die sich für die innere Medizin fruchtbringend erweisen, als dies in den letzten 21/2 Decennien der Fall war, damit der Kongreß für innere Medizin sich weiter entfalte, zum Ruhme der deutschen Medizin und zum Nutzen der leidenden Menschbeit. 